EUROPHYSICS LETTERS

15 September 1998

Europhys. Lett., 43 (6), pp. 664-670 (1998)

\title{
Discontinuous Crack Fronts of Three-Dimensional Fractures
}

\author{
Yoshimi Tanaka ${ }^{1}$, Koji FukaO ${ }^{2}$, Yoshinisa Miyamoto ${ }^{2}$ and Ken Sekimoto ${ }^{3}$ \\ ${ }^{1}$ Graduate School of Human and Environmental Studies, Kyoto University, \\ Kyoto, 606-8501 Japan \\ ${ }^{2}$ Faculty of Integrated Human Studies, Kyoto University, Kyoto, 606-8501 Japan \\ 3 Yukawa Institute for Theoretical Physics, Kyoto University, Kyoto, 606-8502 Japan
}

(received 9 September 1997; accepted in final form 24 July 1998)

PACS. 46.30.Nz - Fracture mechanics, fatigue, and cracks.

PACS. 61.41.+e- Polymers, elastomers, and plastics.

PACS. 62.20.Mk- Fatigue, brittleness, fracture, and crack.

\begin{abstract}
The relation between fracture surface morphology and the three-dimensional structure of crack fronts is investigated through direct observation of brittle cracks in gels. A key notion in this investigation is the discontinuity of the crack front, whose advancement creates the fracture surface. We discuss the significance of our findings in the studies of general three-dimensional brittle fractures.
\end{abstract}

The crack front of a three-dimensional fracture is a one-dimensional object whose advancement into a sample creates a crack. The presence of the third dimension here allows for phenomena which are not seen in two-dimensional fractures [1]. Actual crack fronts are usually not straight lines; many step lines are found on fracture surfaces found in rock [2], glass [3, 4, 5, 6, 6, ceramics [8], rubber [9], gel [10] (including edible jelly), etc. Frechette [8] has pointed out that a crack often becomes an aggregation of crack segments which become interconnected during the course of fracture propagation and thus that such a crack front does not define a continuous line. Theoretically, the emergence of non-planar cracks from the front of planar cracks has been studied by considering sinusoidally varying perturbation applied to a straight crack front 11 , 12 and the 'planting' of planar crack segments ahead of a parent planar crack [13, 14, 15]. Little is known, however, about actual fronts of well-developed cracks 116, 17.

Because of the variety of complicated forms exhibited by general three-dimensional fracture, it would be instructive to begin a study of such phenomena by carrying out a geometrical characterization of the most elementary structures of genuine three-dimensional cracks, just as two-dimensional fractures have been characterized in the terms of elementary mode- $I, I I$, and $I I I$ cracks. Mechanical characterization and molecular theory may follow a geometrical characterization. It is the aim of this paper to present the most elementary geometrical characterization of three-dimensional cracks, based on direct observation. For this purpose, the choices of a suitable material system and investigative method are crucial. Some experiments have been conducted using dense glasses, but in these systems crack growth is very fast, and it is therefore difficult to observe crack fronts in situ.

We have previously reported [10] on pattern formation involving steps which appear on fracture surfaces of gels and on the related dynamical transition with a critical crack growth

Typeset using EURO-LATEX 


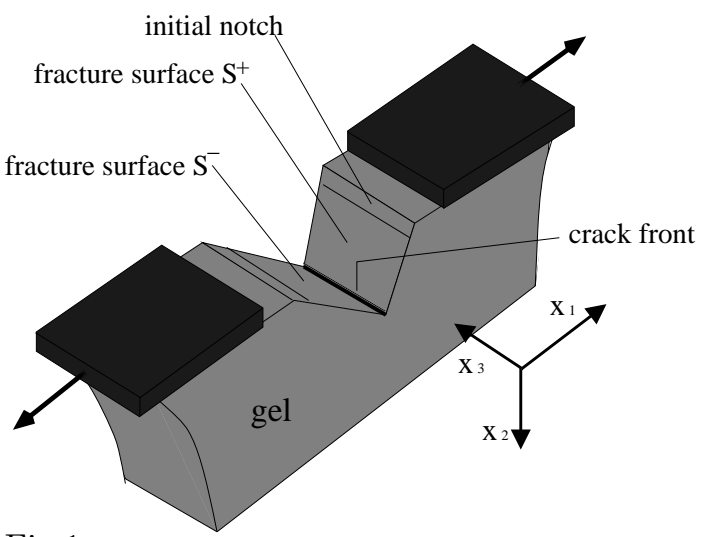

Fig.1
See attached Fig.2 (fig_2.jpg)

Fig. 1. - Schematic depiction of a gel sample undergoing fracture. The Cartesian coordinates are introduced to simplify the discussion given in the text. The sample assumes a pillar-shape of dimension $5 \mathrm{~cm} \times 5 \mathrm{~cm} \times 10 \mathrm{~cm}$. A notch of $3 \mathrm{~mm}$ depth has been introduced transversally to the $x_{1}$-direction. Fracture is induced by moving apart a pair of the plates which are attached to opposite sides of the notch. The crack front propagates in the $+x_{2}$-direction. The crack front is globally parallel to the $x_{3}$-direction and in the plane of $x_{1}=0$. Behind the crack front a pair of fracture surfaces, $S^{+}$and $S^{-}$, are created on the $x_{1}>0$ and $x_{1}<0$ sides, respectively.

Fig. 2. - Global view of a fracture surface $S^{+}$. This photograph was taken after the crack front has passed, from the top to the bottom, through the entire sample. The global speed of the crack front was $0.03 \mathrm{~cm} / \mathrm{sec}$. Step lines are seen as diagonal lines. The bar represents a length of $3 \mathrm{~mm}$.

velocity. There are several notable advantages of using polymer gels for the study of brittle fracture. First, they exhibit no ductile fracture related to dislocation or similar defects around crack fronts. In addition, fracture in a gel proceeds very slowly compared with that in ordinary solid materials. Also, from a technical point of view, the optical transparency of gels facilitates in situ observation of cracks. In this Letter we report the first direct observations of crack fronts in well-developed gel fractures. (There does exist a related study [9], but this is restricted to the initiation of non-planar cracks from a straight crack front. Discussion related to this point is given below.) We elucidate the structure of cracks near discontinuities in crack fronts, and we assert, based on a topological argument, that this structure is a prototype of general three-dimensional brittle fracture.

Our experiment was performed on pillar-shaped gels of dimension $5 \mathrm{~cm} \times 5 \mathrm{~cm} \times 10 \mathrm{~cm}$. The gels were prepared by a standard method using free radical polymerization of acrylamide $(100 \mathrm{mM})$ and bisacrylamide $(8.6 \mathrm{mM})$ initiated by ammonium persulphate and catalyzed by tetramethylethylenediamine [18]. A single notch, 3mm in depth and $0.05 \mathrm{~mm}$ in thickness, was created along a direction transverse to the longest dimension in each sample by placing a stainless-steel sheet in the mold used for polymerization. Samples prepared in this manner were used for the fracture experiments without further treatment. Figure 1 gives a schematic depiction of a gel undergoing fracture, where Cartesian coordinates have been introduced to facilitate the following description. Two plates were attached to each side of the notch, and mechanically driven apart. As the plates spread, the crack front emerged from the initial notch 
See attached color Fig.3 (fig_3.jpg)

Fig. 3. - Configuration of a part of a crack near a discontinuity in the crack front, from which a pair of step lines (one on $S^{+}$and the other on $S^{-}$) extend. a-c show the close-up of the region viewed from the $+x_{1}<0(\mathrm{a}), x_{1}>0(\mathrm{~b})$ and $x_{2}>0(\mathrm{c})$, sides $\mathrm{d}$ is a cross-section of the pair of step lines, viewed from the $x_{2}<0$ side. The crack was almost closed before taking photographs. In order to visualize the fracture surfaces, they have been stained by water-soluble blue paint. The bars in a-c indicate a length of $0.3 \mathrm{~mm}$. e is the stereo view (parallel-viewing) of the region of a crack where a pair of step lines extend. This structure is determined from a-d.

and advanced in the direction of increasing $x_{2}$. During the creation of the fracture, the crack front was globally parallel to the $x_{3}$-direction and in the $x_{1}=0$ plane. Through this process, a pair of fracture surfaces were created behind the crack front. We shall distinguish these fracture surfaces by the symbols $S^{+}$and $S^{-}$, as shown in Fig.1; that is, $S^{+}\left[S^{-}\right.$] represents the fracture surface on the $x_{1}>0\left[x_{1}<0\right]$ side. The crack growth rate (i.e., the global speed of the crack front advancing in the $+x_{2}$-direction in Fig.1) can be controlled through the rate at which the two plates are driven apart. In this study we fixed the crack growth rate at $0.03 \mathrm{~cm} / \mathrm{sec}$. (It is known 10] that morphological features of fracture surfaces are insensitive to crack growth rates below the critical value of dynamical transition, $v_{c} \sim 0.1 \mathrm{~cm} / \mathrm{sec}$.)

Figure 2 is a photograph of a global view of a fracture surface $S^{+}$, taken after a crack has expanded across an entire sample. The many oblique lines on the fracture surface constitute 'steps'. We shall refer to these lines as step lines. During the growth of the fracture surfaces $S^{+}$and $S^{-}$(see Fig.1), many step lines extend simultaneously in a pairwise manner so that each step line on $S^{+}$has a counterpart on $S^{-}$. We carefully observed the region near the front of a crack to determine how a pair of step lines are continuously extended (Figs.3a-d). Figures 3a-c were obtained using the following method. The growth of the crack was halted at an arbitrary instant, and the crack surface was then stained with water-soluble paint. Then, after 
almost closing the crack, we observed through an optical microscope the small region where a pair of step lines meet each other. Figures 3a-c present, respectively, the views from the $x_{1}<0$ (Fig.3a), $x_{1}>0$ (Fig.3b) and $x_{2}>0$ (Fig3.c) sides. Figure 3d shows the cross section of such a pair of step lines, viewed from the $x_{2}<0$ side. This cross section was obtained by cutting the sample along an $x_{2}=$ const. plane, slightly behind (i.e., corresponding to a smaller $x_{2}$-coordinate than) the front of the crack. In Fig.3d, the crack is slightly opened (gap in blue of Fig.3d), and across it a pair of the step lines, one on $S^{+}$(the upper edges of the gap) and the other on $S^{-}$(the lower edges), face each other. This gap has a 'tip-to-line' shape, which resembles an upside-down the Greek letter " $\tau$ ". We represent the configuration of crack creating such a gap by the symbol $\digamma$.

From the above observations, we obtain a geometrical characterization of the position at which a pair of step lines extend (Fig.3e). Here, as in Figs.3a-d, only a small portion of the sample is shown (the frame in black). The crack, drawn in red and blue, is slightly opened. The red curves indicate the crack front, i.e., the front at which the actual irreversible fracture process is taking place. The blue oblique line which runs to $P_{1}$ is not the crack front because no fracture takes place on it. It is the trace of an end point of the crack fronts. (see below descripion.) During the growth of the crack, the crack fronts move obliquely downward, at some acute angles to both the $x_{2}$ - and $x_{3}$ - axes, while keeping the shape of the curves unchanged. As a consequence, the configuration of the fracture surfaces shown in Fig.3e propagates obliquely, from top right to bottom left. In Fig.3e the crack front consists of two discontinuous parts; the left part extending towards (approximately) the negative $x_{3}$-direction has an endpoint at

See attached color Fig.4 (fig_4.jpg)

Fig. 4. - Classification of the intersections: The fracture surface $S^{+}$around each intersection is schematically depicted, together with the cross-section of the step lines related to the intersection and the microscope image in the case of a. The types of $\tau$-structure entering into or emerging from an intersection are identified from these cross-sections. They are indicated by the symbols $J$, etc. These figures show that whether the point-like intersections ( $a$ and $b$ ) or the elongated intersections (c-e) are realized depends on the types of $\tau$-structures entering into the intersections. We do not show those classes of intersections which can be obtained as mirror images (with respect to the $x_{1} x_{2}$-plane and/or the $x_{2} x_{3}$-plane) of the classes shown in a-e. 

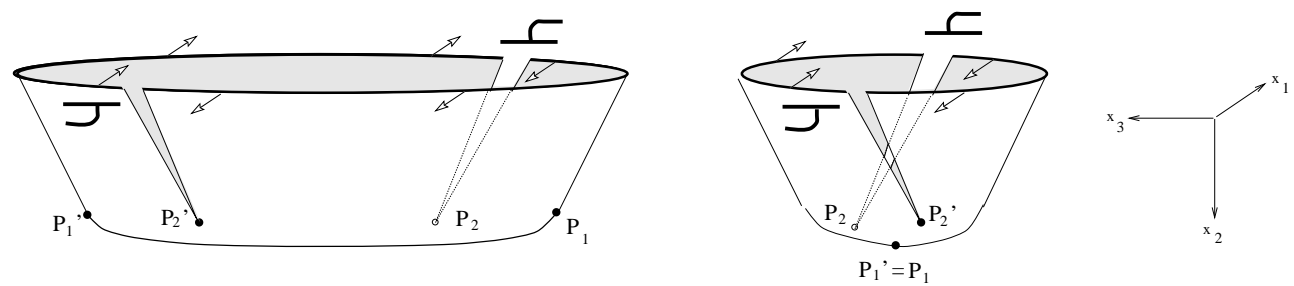

Fig. 5. - Schematic views of the part of a crack involving two step lines of the $\ulcorner$-type and $J$-type. In the top figure, the step lines are separated, where a segment of the crack front (thick curve) is bounded by the points $P_{1}$ and $P_{1}^{\prime}$. In the right figure, $P_{1}$ and $P_{1}^{\prime}$ merge to form an elongated intersection (see Fig.4).

$P_{1}$, while the right part extending toward the positive $x_{3}$-direction has an endpoint $P_{2}$. The endpoint $P_{2}$ is on the left part of the fracture surface, which was created by the advancement of the left part of the crack fronts. We expect this configuration to be quite generic, because it is a topological requirement that the crack be three-dimensionally connected (see below for further discussion).

By symmetry, there are three other variants of the configuration shown in Fig.3. Each of these appears as a mirror image of the form in Fig.3 reflected in the $x_{1} x_{2}$-plane and/or the $x_{2} x_{3}$-plane in the coordinate system of that figure. We will distinguish these four types of configurations by the symbols $\Gamma, \mathcal{工}, \boldsymbol{J}$, and $\tau$. These symbols are caricatures of the hypothetical cross-sections of the respective configurations as appearing in planes parallel to the $x_{1} x_{3}$-plane and viewed from the $x_{2}<0$ side, as shown in Fig.3d (see also Figs.4a-e and Fig.5). We will refer to the portion of a crack characterized by these four kinds of configurations as $\tau$-structure. When cracks grow under global crack-opening forces along the $\pm x_{1}$-directions, all four types of $\tau$-structure mentioned above occur with equal probability.

An intersection is formed when a $\tau$-structure of either type $\mathcal{工}$ or $\mathcal{J}$ meet with another $\tau$-structure of either type $\tau$ or $\boldsymbol{\Gamma}$. Figures 4 a-e represent the intersections of the step lines created on a crack surface, $S^{+}$. An intersection which appears as a simple crossing of two straight lines (a point-like intersection for short) occurs if the two $\tau$-structures meeting in this manner are combinations of either types $\boldsymbol{J}$ and $\tau$ (Fig.4a and Fig.4b), or $\mathcal{I}$ and $\Gamma$. On the other hand, an intersection that contains a short line element virtually parallel to the $x_{2}$-axis (a prolonged intersection for short) occurs for a combination of either types $\beth$ and $\tau$ (Fig.4c, Fig.4d and Fig4.e), or $\mathcal{\Gamma}$ and $\mathcal{J}$. In the latter case, real-time observation revealed the crack growth was temporarily arrested at the point where the two types of $\tau$-structure met. Figure 5 schematically displays what should occur for the crack during this period; the point $P_{2}$ of $ᄃ$ and its counterpart $P_{2}^{\prime}$ of $J$ are on opposite sides of the opening crack. As the $x_{3}$-coordinate of $P_{2}$ becomes larger than that of $P_{2}^{\prime}$ (the right figure in Fig.5), the global mode- $I$ loading (the

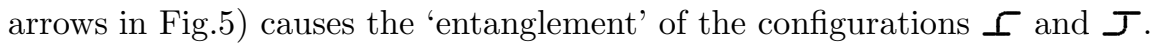

Figure 4 shows that, given the types of $\tau$-structure entering an intersection, the types of $\tau$-structure emerging from the intersection are restricted but not uniquely determined. In order to solve this selection problem, we would need to know the distribution of strain or stress 16] in the sample. The strain or stress near a $\tau$-structure should in turn depend on the presence of other $\tau$-structures and intersections, as well as the possible existence of inhomogeneity in the gel 19. We expect from symmetry considerations that the $x_{2} x_{3}$ component of shear stress, $T_{23}$, and the $x_{1} x_{3}$ component (i.e. 'mode-III loading') will affect the four types of $\tau$-structures

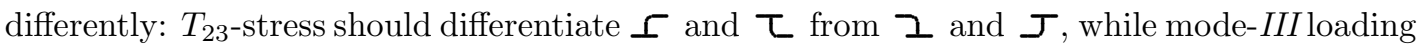




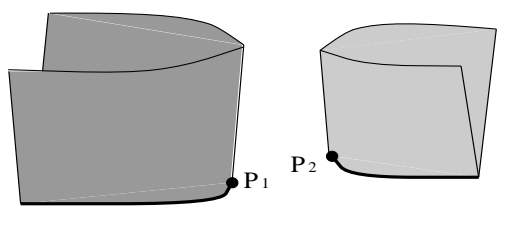

(a)

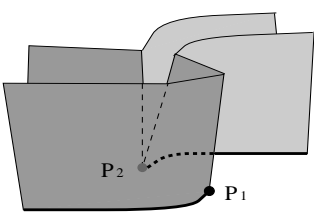

(b)

Fig. 6. - (a) Hypothetical cracks created by a discontinuous crack front. These two disconnected cracks would not divide the sample into two pieces. (b) The two cracks, being connected, make a continuous crack despite the presence of a discontinuous crack front. The resulting crack structure is equivalent to that shown in Fig.3.

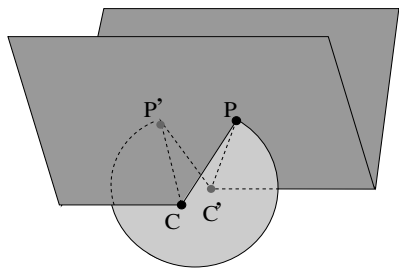

(a)

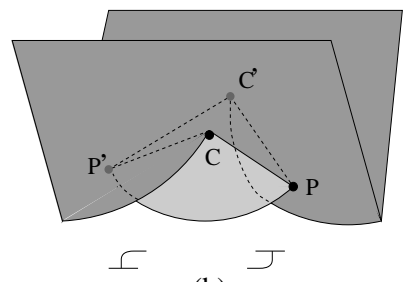

(b)

Fig. 7. - Illustration of the notion of the homology between two types of crack structures. The structure $(a)$ can be transformed into $(b)$ by a continuous deformation, pulling down the points $P$ and $P^{\prime}$, while raising up the points $C$ and $C^{\prime}:(a)$ is an elementary structure of so-called parahelical cracks [9]. The dark portion is the parent crack, and the light portion is a helical crack, which emerges from the parent crack. The points $P$ and $P^{\prime}$ (the counterpart of $P_{2}$ in Figs. 1 and 5) are on opposite sides of the parent fracture surface. (b) illustrates the nucleation of a pair of structures, $\digamma$ and $\mathcal{J}$, being juxtaposed in this order.

should differentiate $\Gamma$ and $J$ from $工$ and $\tau$.

The $\tau$-structures described above should be ubiquitous in the fracture of three-dimensional materials because the $\tau$-structures embody the most generic feature of discontinuous crack fronts as discussed below. The most important point in this discussion is that in the process of breaking a three-dimensional material into two pieces, a fracture surface must always be continuous. The question we must address is the following: what topologies of cracks allow for the realization of both the discontinuity of the crack front and the continuity of the fracture surface? To answer this in a generic manner, allow us to consider the situation in which, starting with two hypothetical partial fracture surfaces created by two arbitrary discontinuous crack fronts (Fig.6a), we connect the partial fracture surfaces in the manner shown in Fig.6b. The case depicted here corresponds to the $\tau$-structure $\mathcal{\Gamma}$, and this structure along with its three variants constitute all the generic ways of connecting the two partial fracture surfaces shown in Fig.6a.

We may find as an element the topological object characterized by $\tau$-structure in any fracture surface that consists of more than one part. For example, in a swollen elastomer [9], an array of helical crack segments (see Fig.7a) appears on the rim of a parent planar crack (called a parahelical crack [9]). Each helical crack can be continuously deformed into the juxtaposition of $\Gamma$ and $\boldsymbol{J}$ (or of $\tau$ and $\boldsymbol{工}$ ), as shown in Fig.7b. The points $P$ and $P^{\prime}$ in Fig.7 correspond to $P_{2}$ in Figs. 3 and 5.

Before concluding, we note a polymer physics aspect of the fracture of gels. The cooperative 
diffusion should be induced in the largely deformed region 20 near a crack front. The length scale $D_{\text {coop }} / V_{c}$, where $D_{\text {coop }}\left(\sim 10^{-7} \mathrm{~cm}^{2} / \mathrm{sec}\right)$ is the cooperative diffusion constant 21] and $V_{c}(\sim 0.1 \mathrm{~cm})$ is the measured critical crack growth rate of the dynamical transition [10], is just on the order of the blob size $\xi(\sim 100 \AA)$ [22]. This suggests macroscopic features of the fracture of gels change depending on whether or not 'diffusion length' $D_{\text {coop }} / V$ exceeds the blob size of gels.

In this Letter, we have reported the direct observation of crack fronts in slow, brittle fracture of gels. The structure near the discontinuity of the crack front was elucidated, and its implications were discussed from a topological viewpoint. Such a topological viewpoint of three-dimensional fracture should be helpful in experimental, numerical and theoretical studies, for which a gel can serve as a suitable model system.

\section{$* * *$}

The authors thank Yoshi Oono, Albert Libchaber and an anonymous referee for critical comments on the earlier version of the manuscript. The work was partly supported by a Grant-in-Aid from the Ministry of Education, Science, Sports and Culture of Japan.

\section{REFERENCES}

[1] Recent developments in 2D fractures can be found in; Grimson, M. J., Molecul. Phys., 76 (1992) 1375, Freund, L. B., 'Dynamic Fracture Mechanics' (Cambridge Univ. Press, New York,1990), Sasa, S., Sekimoto, K. and Nakanishi, H., Phys. Rev. E, 50 (1994) R1733, Ching, E. S. C., Langer, J. S. and Nakanishi, H., Phys. Rev. Lett., 76 (1996) 1087, and the references cited therein.

[2] Pollard, D. D. and Aydin, A. Geol. Soc. America Bulletin, 100 (1988) 1181.

[3] Sommer, E., Eng. Fracture Mech., 1 (1969) 539.

[4] Abdel-Latif, A. I. A., Bradt, R. C. and Tressler, R. E., Int. J. Fracture, 13 (1977) 349.

[5] Döll, W., "Fractography and failure mechanisms of polymers and composites", ed. by A. C. Roulin-Moloney (Elsevier Science Pub., London and New York, 1989) Chap. 10, pp. 387-436.

[6] Chevrier, J., J. Phys. I France, 5 (1995) 675.

[7] Beauchamp, Edwin k. J. Am. Ceram. Soc. 78 [3] 689 (1995).

[8] Frechette,V.D. Advances in Ceramics, 28 (1990) 21.

[9] Palaniswamy, K. and Knauss, W. G., Mechanics Today, 4 (1978) 87.

[10] Tanaka, Y., Fukao, K., Miyamoto, Y., Nakazawa, H. and Sekimoto, K., J. Phys. Soc. Japan, 65 (1996)2349.

[11] Gao, H., J. Appl. Mech., 59 (1992) 335.

[12] Xu, G., Bower, A. F. and Ortiz. M., Int. J. Solids Structures, 31 (1994) 2167.

[13] Lawn, B. R. and Wilshaw, T. R., 'Fracture of brittle solids' (Cambridge Univ. Press, Cambridge, UK, 1975); Lawn, B. R. ibid. 2nd Edition, 1993.

[14] Pollard, D. D., Segall, P. and Delaney, P. T., Geol. Soc. America Bull., 93 (1982) 1291.

[15] Gell, M. and Smith, E., Acta Metallurgica, 15 (1967) 253.

[16] Chaudhri, M.M. and Liangyi, C., Nature, 320 (1986) 48.

[17] Marder, M., Nature (News and Views), 381 (1996) 275, and the reference cited therein.

[18] Tanaka, T., Phys. Rev. Lett., 40 (1978) 820.

[19] Bastide, J. and Leibler, L., Macromolecules, 21 (1990) 1821.

[20] Suzuki, A., Sanda, K. and Omori, Y., J. Chem. Phys., 107 (1997) 5179.

[21] De Gennes, P.-G., Scaling Concepts in Polymer Physics (Cornell Univ. Press, New York, 1979).

[22] Cohen, Y., Ramon, O., Kopelman, I.J. and Mizrahi, S., Journal of Polymer Science:Part B, 30 (1992) 1055. 
This figure "fig_2.jpg" is available in "jpg" format from: http://arxiv.org/ps/cond-mat/9810139v1 
This figure "fig_3.jpg" is available in "jpg" format from: http://arxiv.org/ps/cond-mat/9810139v1 
This figure "fig_4.jpg" is available in "jpg" format from: http://arxiv.org/ps/cond-mat/9810139v1 